\title{
A family of geometric operators on triangles with two complex variables
}

\author{
HIROAKI NAKAMURA AND HIROYUKI OGAWA
}

\begin{abstract}
Given a plane triangle $\Delta$, one can construct a new triangle $\Delta^{\prime}$ whose vertices are intersections of two cevian triples of $\Delta$. We extend the family of operators $\Delta \mapsto \Delta^{\prime}$ by complexifying the defining two cevian parameters and study its rich structure from arithmeticgeometric viewpoints. We also find another useful parametrization of the operator family via finite Fourier analysis and apply it to investigate area-preserving operators on triangles.
\end{abstract}

\section{Introduction}

In [7, we studied a certain operator $\mathcal{S}_{p, q}$ on plane triangles with two real parameters $p, q$ $\left(p q \neq 1,(p, q) \neq\left(\frac{1}{2}, \frac{1}{2}\right)\right)$ which maps any triangle $\Delta A B C$ to a new triangle $\Delta A^{\prime} B^{\prime} C^{\prime}$ such that the vertex $A^{\prime}$ (resp. $B^{\prime}, C^{\prime}$ ) is obtained as the intersection of the $(p: 1-p)$-cevian from the vertex $B$ (resp. $C, A)$ and the $(1-q: q)$-cevian from the vertex $A$ (resp. $B, C)$.

The aim of this paper is to extend $\mathcal{S}_{p, q}$ 'algebraically' for complex parameters $p, q$ and to study basic properties of the total collection of those operators. Although our extension differs from geometrically obvious one via generalized cevians, we still can give geometrical interpretation of $\mathcal{S}_{p, q}$ for complex $p, q$ (in Remark 3.6).

After establishing setup and basic properties in $\S 2-3$, we introduce in $\S 4$, a moduli parameter $\xi_{p, q}$ which controls the effect of $\mathcal{S}_{p, q}$ on the shapes (similarity classes) of triangles. In $\S 5$, it turns out that finite Fourier analysis enables us to find natural separation of numerator and denominator of $\xi_{p, q}$ in the form $\xi_{p, q}=\eta_{p, q} / \eta_{p, q}^{\prime}$, which leads us to introduce a more natural family of operators $\mathcal{S}\left[\eta, \eta^{\prime}\right]$ defined all over the pairs $\left(\eta, \eta^{\prime}\right) \in \mathbb{C}^{2}$. We give explicit expression of the original parameters $p, q$ as rational functions in $\eta, \eta^{\prime}$. Finally in $\S 6$, we study "area-preserving normal operators" parametrized by a standard torus $S^{1} \times S^{1}$ in $\mathbb{C}^{2}$

Throughout this paper, we use the notations: $\rho:=e^{2 \pi i / 6}, \omega:=e^{2 \pi i / 3}$.

\section{Operators $\mathcal{S}_{p, q}$}

A triangle is a set $\{a, b, c\}$ of three points (vertices) on the complex plane $\mathbb{C}$. We will consider any ordered triple $(a, b, c) \in \mathbb{C}^{3}$ with distinct coordinates as a triangle triple representing $\{a, b, c\}$. It is said to be degenerate if $a, b, c$ are collinear. A non-degenerate triangle triple $(a, b, c)$ is said to be positive (resp. negative) if it represents a triangle $\{a, b, c\}$ with $(a-b) /(c-b)$ belonging to the upper half plane $\mathcal{H}^{+}=\{z \in \mathbb{C} \mid \operatorname{Im}(z)>0\}$ (resp. the lower half plane $\left.\mathcal{H}^{-}=\{z \in \mathbb{C} \mid \operatorname{Im}(z)<0\}\right)$.

In this paper, we study operations $\mathcal{S}_{p, q}(p, q \in \mathbb{C}, p q \neq 1)$ on the triangle triples defined by

$$
\mathcal{S}_{p, q}(a, b, c)=\left(a^{\prime}, b^{\prime}, c^{\prime}\right):\left\{\begin{array}{l}
a^{\prime}=\alpha_{p, q} a+\beta_{p, q} b+\gamma_{p, q} c \\
b^{\prime}=\alpha_{p, q} b+\beta_{p, q} c+\gamma_{p, q} a \\
c^{\prime}=\alpha_{p, q} c+\beta_{p, q} a+\gamma_{p, q} b
\end{array}\right.
$$


where,

$$
\alpha_{p, q}=\frac{p(1-q)}{1-p q}, \beta_{p, q}=\frac{q(1-p)}{1-p q}, \gamma_{p, q}=\frac{(1-p)(1-q)}{1-p q} .
$$

We will often identify $\mathcal{S}_{p, q}$ with its obvious extension to the linear operator on $\mathbb{C}^{3}$ defined by the same formula (2.1). The above operator $\mathcal{S}_{p, q}$ is designed to preserve centroids of triangles due to the condition $\alpha_{p, q}+\beta_{p, q}+\gamma_{p, q}=1$ (cf. Proposition 4.4).

For real parameters $(p, q)$, the operator $\mathcal{S}_{p, q}$ was studied in [7] where $a^{\prime}, b^{\prime}, c^{\prime}$ are given as the intersection points of certain cevians of $\{a, b, c\}$ determined by $(p, q)$. In [4], T.Kanesaka inspected $\mathcal{S}_{p, q}$ extended to complex variables $(p, q)$ in the above form. In Remark 3.6, we will discuss a geometrical interpretation of $\mathcal{S}_{p, q}$ for complex $p, q$ which uses more tools than cevians.

Proposition 2.2. Let $p, q \in \mathbb{C}$ satisfy $p q \neq 1$. The following two conditions are equivalent.

(i) $\mathcal{S}_{p, q}$ maps every positive triangle triple to a positive triangle triple.

(ii) $p=q \neq \frac{1}{2}$ or $\frac{(p-1)(2 q-1)}{p-q} \in \mathbb{R} \cup \mathcal{H}^{+}$.

Proof. Given a positive triangle triple $(a, b, c)$, let $\mathcal{S}_{p, q}(a, b, c)=\left(a^{\prime}, b^{\prime}, c^{\prime}\right)$. For $z=(a-b) /(c-$ b) $\in \mathcal{H}^{+}$, one computes:

$$
F(z)=\frac{a^{\prime}-b^{\prime}}{c^{\prime}-b^{\prime}}=\frac{(1-q)(2 p-1) z+(1-p)(1-2 q)}{(1-p)(2 q-1) z+(p-q)} .
$$

The condition (i) is equivalent to that the mapping $z \mapsto F(z)$ defines a linear fractional transformation sending $\mathcal{H}^{+}$into $\mathcal{H}^{+}$. When $p=q,(p, q) \neq\left(\frac{1}{2}, \frac{1}{2}\right)$ rephrases non-degeneracy of $\left\{a^{\prime}, b^{\prime}, c^{\prime}\right\}$, in which case $F(z)=\frac{z-1}{z}$ maps $\mathcal{H}^{+}$onto $\mathcal{H}^{+}$certainly. When $p \neq q$, writing $t:=(p-1)(2 q-1) /(p-q)$, one finds

$$
F(z)=\frac{(t-1) z-t}{t z-1}
$$

which represents a non-degenerate linear fractional map with $F(\rho)=\rho$ if and only if $t \neq \rho$. In this case, the condition (i) is equivalent to $F(0)=t \in \mathbb{R} \cup \mathcal{H}^{+}$. When $t=\rho$, constantly $F(z)=\rho$ so that $\mathcal{S}_{p, q}$ maps every positive triangle triple to a positive equilateral triangle triple.

Definition 2.3. The operator $\mathcal{S}_{p, q}$ will be called regular if the condition of Proposition 2.2 is satisfied. Moreover, we shall call $\mathcal{S}_{p, q}$ to be normal, if it is regular with $(p-1)(2 q-1) /(p-q) \in \mathbb{R}$.

Example 2.4. Let $\Delta=(a, b, c)$ denote a triangle triple. In [7], we presented two major examples $\mathcal{S}_{0, q}$ and $\mathcal{S}_{1-q, q}$ for $q \in \mathbb{R}$. $\mathcal{S}_{0, q}(\Delta)$ is a triangle triple whose vertices are the " $(1-q): q$ "-division points of sides of $\Delta$, while $\mathcal{S}_{1-q, q}(\Delta)$ is a triangle triple obtained from the " $(1-q): q$ "-cevians. In [3], these are studied under the name of $s$-medial and $s$-Routh triangles $\mathcal{M}_{s}(\Delta), \mathcal{R}_{s}(\Delta)$ which, in our notations, correspond to $\mathcal{S}_{0,1-s}(\Delta)$ and $\mathcal{S}_{s, 1-s}(\Delta)$ respectively. There is another interesting construction called the $s$-median triangle of $\Delta$ (written $\mathcal{H}_{s}(\Delta)$ in [3]). In [6], we discuss a generalization of $\mathcal{H}_{s}(\Delta)$ from the viewpoint of the present paper.

Example 2.5. The famous Napoleon's theorem tells that, for any triangle $\Delta$, the centers of the three equilateral triangles sharing one of the sides of $\Delta$ form an equilateral triangle. This means, for $q=\frac{1-\omega^{2}}{3}=\frac{1}{2}+\frac{\sqrt{3} i}{6}, \mathcal{S}_{0, q}(\Delta)$ is equilateral for every triangle triple $\Delta$. This $\mathcal{S}_{0, q}$ gives a regular but a non-normal operator which motivates us to investigate complex parameters $(p, q)$.

\section{Matrix analysis of $\mathcal{S}_{p, q}$}

In this section, we summarize basic facts on $\mathcal{S}_{p, q}$ from the 3 by 3 matrix point of view. Let

$$
\mathrm{I}:=\left(\begin{array}{ccc}
1 & 0 & 0 \\
0 & 1 & 0 \\
0 & 0 & 1
\end{array}\right), \quad \mathrm{J}:=\left(\begin{array}{ccc}
0 & 1 & 0 \\
0 & 0 & 1 \\
1 & 0 & 0
\end{array}\right)
$$


and define

$$
\mathrm{S}_{p, q}:=\alpha_{p, q} \mathrm{I}+\beta_{p, q} \mathrm{~J}+\gamma_{p, q} \mathrm{~J}^{2}
$$

From 2.1) follows that

$$
\mathcal{S}_{p, q}(a, b, c)=\mathrm{S}_{p, q}\left(\begin{array}{l}
a \\
b \\
c
\end{array}\right)
$$

where triangle triples $(a, b, c)$ is regarded as column vectors in $\mathbb{C}^{3}$. It is natural to identify $\mathcal{S}_{p, q}$ with $\mathrm{S}_{p, q} \in \mathrm{GL}_{3}(\mathbb{C})$ acting on the column vector space $\mathbb{C}^{3}$ on the left. It is easy to see that

$$
\mathrm{S}_{0,0}=\mathrm{J}^{2} ; \quad \mathrm{S}_{1, q}=\mathrm{I} \quad(q \neq 1) ; \quad \mathrm{S}_{p, 1}=\mathrm{J} \quad(p \neq 1)
$$

and that $\mathrm{S}_{1,1}$ is essentially undefined ( $p=q=1$ is a point of indeterminacy). Observe that the operators $\mathcal{S}_{0,0}, \mathcal{S}_{1, q}(q \neq 1)$ induce cyclic permutations on the vertices of triangle triples.

Definition 3.3. We call $\mathcal{S}_{0,0}, \mathcal{S}_{p, 1}(p \neq 1)$ cyclic permutation operators, and call $\mathcal{S}_{1, q}(q \neq 1)$ identity operators.

Example 3.4. On the other hand, there are no $\mathcal{S}_{p, q}$ that universally induce transpositions on vertices of triangle triples. However, given an individual triangle triple $(a, b, c)$, one finds

$$
\begin{aligned}
& \mathcal{S}_{p, q}(a, b, c)=(a, c, b) \quad \text { for }(p, q)=\left(\frac{c-a}{b-a}, \frac{a-c}{b-c}\right), \\
& \mathcal{S}_{p, q}(a, b, c)=(b, a, c) \quad \text { for }(p, q)=\left(\frac{b-c}{a-c}, \frac{c-b}{a-b}\right), \\
& \mathcal{S}_{p, q}(a, b, c)=(c, b, a) \quad \text { for }(p, q)=\left(\frac{a-b}{c-b}, \frac{b-a}{c-a}\right) .
\end{aligned}
$$

These are called reflection operators for the triangle triple $\Delta=(a, b, c)$ and form first basic examples of non-regular $\mathcal{S}_{p, q}$. It is a good exercise to show that $\mathcal{S}_{p, q}$ is a reflection operator for some $\Delta$ if and only if the parameters $p, q \in \mathbb{C}$ satisfy $p q \neq 1$ and $p q=p+q$. (Hint: We have $\mathcal{S}_{p, q}(a, b, c)=(a, c, b)$ if $\left.a=(1-q) c+q b.\right)$

Below we first prepare a notion of weighted mean (average) of two matrices (also of two vectors), which will be used in the subsequent Remark.

Definition 3.5. Given a complex number $r \in \mathbb{C}$ and two matrices $A, B$ of a same size, we define the $r$-weighted mean, or for short, $r$-average of $A$ and $B$, by $\mu_{r}(A, B):=(1-r) A+r B$.

Remark 3.6. (Geometrical interpretation.) In [8, p.76, Remark], G.Nicollier gave a useful geometrical interpretation of the operator $\mathcal{S}_{p, q}$ in terms of convolution with generalized Kiepert triangle ([8, p.69]. See also proof of Proposition 5.1 below.)

Here, we give another interpretation in terms of consecutive operations of weighted arithmetic means of triangles. It follows from a simple matrix calculation that

$$
\mathrm{S}_{p, q}=\mu_{\frac{1-q}{1-p q}}\left(\mathrm{~J}, \mu_{p}\left(\mathrm{~J}^{2}, \mathrm{I}\right)\right)=\mu_{\frac{1-p}{1-p q}}\left(\mathrm{I}, \mu_{q}\left(\mathrm{~J}^{2}, \mathrm{~J}\right)\right) .
$$

Geometrical interpretation of the above identity applied to a triangle triple $\Delta=(a, b, c)$ may be phrased as follows. First, form the $p$-average $\Delta^{\prime}=\mu_{p}\left(\mathrm{~J}^{2} \Delta, \Delta\right)$ of 'label-permuted' triangles $\mathrm{J}^{2} \Delta=(c, a, b)$ and $\Delta=(a, b, c)$. Then, one obtains $\mathcal{S}_{p, q}(\Delta)$ as the $\frac{1-q}{1-p q}$-average of $\mathrm{J} \Delta=(b, c, a)$ and $\Delta^{\prime}$. In another way, form the $q$-average $\Delta^{\prime \prime}=\mu_{q}\left(\mathrm{~J}^{2} \Delta, \mathrm{J} \Delta\right)$ of $\mathrm{J}^{2} \Delta=(c, a, b)$ and $\mathrm{J} \Delta=$ $(b, c, a)$. Then, $\mathcal{S}_{p, q}(\Delta)$ is obtained as the $\frac{1-p}{1-p q}$-average of $\Delta=(a, b, c)$ and $\Delta^{\prime \prime}$.

Note 3.8. Generically, it holds that

$$
\mathrm{S}_{p_{2}, q_{2}} \mathrm{~S}_{p_{1}, q_{1}}=\mathrm{S}_{p_{1}, q_{1}} \mathrm{~S}_{p_{2}, q_{2}}=\mathrm{S}_{p, q}
$$


with

where

$$
p=\frac{\lambda_{2}+2 \lambda_{3}}{\lambda_{1}}, \quad q=\frac{\lambda_{1}-2 \lambda_{3}}{\lambda_{2}},
$$

$$
\begin{aligned}
& \lambda_{1}=1-\left(1-2\left(1-p_{1}\right)\left(1-p_{2}\right)\right)\left(1-2\left(1-q_{1}\right)\left(1-q_{2}\right)\right), \\
& \lambda_{2}=1-2\left(1-p_{1} p_{2}\right)-\left(1-2\left(1-p_{1}\right) q_{2}\right)\left(1-2\left(1-p_{2}\right) q_{1}\right), \\
& \lambda_{3}=\left(1-p_{1} q_{1}\right)\left(1-p_{2} q_{2}\right) .
\end{aligned}
$$

But the collection $\mathbb{S}_{p, q}:=\left\{\mathrm{S}_{p, q} \mid p, q \in \mathbb{C}, p q \neq 1\right\}$ is not closed under multiplication in $\mathrm{GL}_{3}(\mathbb{C})$. For example, $\left(p_{1}, q_{1}\right)=\left(\frac{1}{3}, \frac{1}{4}\right),\left(p_{2}, q_{2}\right)=\left(-\frac{7}{8},-\frac{1}{9}\right)$ implies $\lambda_{1}=\lambda_{2}+2 \lambda_{3}=0$ which shows essential indeterminacy of $p$ in this special case. Meanwhile, if $\left(p_{1}, q_{1}\right)=\left(\frac{1}{3}, \frac{1}{4}\right),\left(p_{2}, q_{2}\right)=$ $\left(-\frac{2}{7}, \frac{1}{5}\right)$, then $\lambda_{1}=0$ while $\lambda_{2}+2 \lambda_{3} \neq 0$. In this case, one would be able to regard $p=\infty$ with suitable interpretation about $\mathcal{S}_{p_{1}, q_{1}} \mathcal{S}_{p_{2}, q_{2}}=\mathcal{S}_{\infty, q}$. Later in $\S 5$, we will remedy this inconvenience by extending the set $\mathbb{S}_{p, q}$ to a multiplicative submonoid of $M_{3}(\mathbb{C})$. Cf. Remark 5.11 .

\section{Actions on the moduli disk $\mathcal{D}$}

In [7], we introduced a moduli space of the similarity classes of triangles as the Poincaré disk $\mathcal{D}=\{z \in \mathbb{C} ;|z|<1\}$ by associating with any triangle $\Delta$ the well-defined modulus

$$
\phi(\Delta):=\left(\frac{a+b \omega+c \omega^{2}}{a+b \omega^{2}+c \omega}\right)^{3} \in \mathcal{D},
$$

when $\Delta=\{a, b, c\}$ is labeled by a positive triangle triple $(a, b, c)$. Now, let us introduce two quantities $t_{p, q}$ and $\xi_{p, q}$ by

$$
t_{p, q}:=\frac{(p-1)(2 q-1)}{p-q}, \quad \xi_{p, q}:=\frac{1+t_{p, q} \omega}{1+t_{p, q} \omega^{2}}=\frac{(p-q)+(p-1)(2 q-1) \omega}{(p-q)+(p-1)(2 q-1) \omega^{2}} .
$$

It is not difficult to see that $\mathcal{S}_{p, q}$ is regular (Definition 2.3 if and only if $\left|\xi_{p, q}\right| \leq 1$. Indeed, each regular operation $\mathcal{S}_{p, q}$ induces a moduli action $T_{p, q}: \mathcal{D} \rightarrow \mathcal{D}$ satisfying $T_{p, q}(\phi(\Delta))=\phi\left(\mathcal{S}_{p, q}(\Delta)\right)$ for all triangles $\Delta$ given by the formula

$$
T_{p, q}(z)=\xi_{p, q}^{3} \cdot z
$$

Thus, the moduli action $T_{p, q}$ induced from a regular operator $\mathcal{S}_{p, q}$ maps the moduli disk $\mathcal{D}=$ $\{z \in \mathbb{C} ;|z|<1\}$ into a smaller or the same disk $\left\{z \in \mathbb{C} ;|z|<\left|\xi_{p, q}\right|^{3}\right\}$. In particular, a normal operator $\mathcal{S}_{p, q}$ gives rise to $T_{p, q}$ that rotates $\mathcal{D}$ with angle $\operatorname{Arg}\left(\xi_{p, q}^{3}\right)$ in the anti-clockwise way. It is also reasonable to call such $T_{p, q}$ regular (resp. normal) when $\operatorname{Im}\left(t_{p, q}\right) \geq 0 \Leftrightarrow\left|\xi_{p, q}\right| \leq 1$ (resp. $\left.\operatorname{Im}\left(t_{p, q}\right)=0 \Leftrightarrow\left|\xi_{p, q}\right|=1\right)$.

Here, we summarize basic properties of $\mathcal{S}_{p, q}$, some of which are consequences of (4.3). Write $\bar{\Delta}$ for the complex conjugate of a triangle triple $\Delta$.

Proposition 4.4. $\quad$ (i) If $\mathcal{S}_{p, q}(\Delta)=\Delta^{\prime}$ then $\mathcal{S}_{\bar{p}, \bar{q}}(\bar{\Delta})=\overline{\Delta^{\prime}}$, and vice versa holds.

(ii) The operator $\mathcal{S}_{p, q}$ preserves centroids of triangles, and maps equilateral triangle triples to equilateral triangle triples.

(iii) Let $\Delta$ be an arbitrary triangle triple, and $r, q \in \mathbb{C}, r \neq 2, q \neq-1$. Then, all of $\mathcal{S}_{\frac{1}{2}, r}(\Delta)$, $\mathcal{S}_{r, \frac{1}{2}}(\Delta)$ and $\mathcal{S}_{q, q}(\Delta)$ are similar to $\Delta$.

Proof. (i) follows immediately from the definition of $\mathcal{S}_{p, q}$. (ii) The first assertion is easy to see from $a+b+c=a^{\prime}+b^{\prime}+c^{\prime}$ in (2.1) due to the identity $\alpha_{p, q}+\beta_{p, q}+\gamma_{p, q}=1$ encoded in our definition. For the second, suppose first that $\Delta$ is given as a positive triangle triple representing an equilateral triangle. The action of $\mathcal{S}_{p, q}$ on the similarity class of $\Delta$ is reduced to the moduli operator $T_{p, q}$ acting on the moduli disc through multiplication by $\xi_{p, q}^{3}$. In particular, it fixes 0 
which is the modulus of the equilateral triangles. If $\Delta$ is given as a negative equilateral triple, then $\bar{\Delta}$ is a positive equilateral triple, which, by the above argument, is sent to an equilateral triple by $\mathcal{S}_{\bar{p}, \bar{q}}$. Then, apply (i) to get the asserted conclusion. (iii) Again by virtue of (i), it suffices to show the assertion for positive triangle triples. Direct computation shows that $\xi_{\frac{1}{2}, r}^{3}=\xi_{r, \frac{1}{2}}^{3}=\xi_{q, q}^{3}=1$. It follows from 4.3 that the corresponding operators $\mathcal{S}_{\frac{1}{2}, r}(\Delta), \mathcal{S}_{r, \frac{1}{2}}(\Delta)$ and $\mathcal{S}_{q, q}(\Delta)$ do not change the similarity classes of triangles.

Observe in 4.2 , the quantities $t_{p, q}$ and $\xi_{p, q}$ make senses to be valued in $\mathbf{P}^{1}(\mathbb{C})=\mathbb{C} \cup\{\infty\}$ as long as $(p, q) \neq(1,1),\left(\frac{1}{2}, \frac{1}{2}\right)$ (even when $p q=1$ with $\mathcal{S}_{p, q}$ undefined). Therefore, at the level of moduli action, we shall extend $T_{p, q}$ for all complex pairs $(p, q) \neq(1,1),\left(\frac{1}{2}, \frac{1}{2}\right)$. Consider now the bijective mapping

$$
\psi: \mathbf{P}_{t}^{1}(\mathbb{C}) \stackrel{\sim}{\rightarrow} \mathbf{P}_{\xi}^{1}(\mathbb{C}): t \mapsto \xi=\frac{1+t \omega}{1+t \omega^{2}}
$$

of the (Riemann) $t$-sphere onto the $\xi$-sphere modelled on 4.2$)$, which in particular maps $\psi(0)=$ $1, \psi(\rho)=0$ and $\psi\left(\rho^{-1}\right)=\infty$. Recall here the notations $\rho:=e^{2 \pi i / 6}, \omega:=e^{2 \pi i / 3}$.

Definition 4.5. Let $T(\mathbb{C}):=\mathbf{P}_{t}^{1}(\mathbb{C})-\left\{\rho, \rho^{-1}\right\}$ and introduce a commutative group structure $[+]$ by pulling back the multiplication on $\mathbf{G}_{m}(\mathbb{C})=\mathbf{P}_{\xi}^{1}(\mathbb{C})-\{0, \infty\}$ via $\psi$.

Proposition 4.6. Notations being as above, the following assertions hold:

(i) For $t, t^{\prime} \in T(\mathbb{C}), t[+] t^{\prime}=\frac{t+t^{\prime}-t t^{\prime}}{1-t t^{\prime}}$.

(ii) If $[N]: T(\mathbb{C}) \rightarrow T(\mathbb{C})$ denotes the multiplication by $N$ in the sense of $[+]$ for $N \in \mathbb{Z}$, then

$$
[N] \cdot t=\frac{(1+\omega t)^{N}-\left(1+\omega^{-1} t\right)^{N}}{\rho(1+\omega t)^{N}-\rho^{-1}\left(1+\omega^{-1} t\right)^{N}} .
$$

(iii) The $N$-division points of $T(\mathbb{C})$ are given by

$$
t=\frac{\sin \pi \frac{k}{N}}{\sin \pi\left(\frac{k}{N}+\frac{1}{3}\right)} \quad(k=0,1, \ldots, N) .
$$

Proof. These formulae follow in straightforward ways from the above definitions after noting $\psi\left(t[+] t^{\prime}\right)=\psi(t) \psi\left(t^{\prime}\right), \psi([N] t)=\psi(t)^{N}$ and ' $\psi([N] t)=1 \Leftrightarrow t$ is an $N$-division point'.

The above group structure on $T$ is essentially same as what was introduced in Komatsu [5] and Ogawa [9] for a twisted Kummer theory designed to control $n$-cyclic extensions of number fields containing $\cos (2 \pi i / n)$. (Our case is a special case of $n=6$ with $t^{-1}$ corresponding to the standard coordinate in their theory). Bényi-Ćurgus [2] studied the same additive structure on the 'normal' locus $T(\mathbb{R})$.

In fact, $T$ has a natural structure of algebraic torus defined over $\mathbb{Q}$ : one can equip $T$ with the $\mathbb{Q}$-structure of the affine conic (ellipse) $S: u^{2}+u v+v^{2}=1$ by transformations by

$$
u=\frac{1-t^{2}}{t^{2}-t+1}, v=\frac{t(t-2)}{t^{2}-t+1} ; \quad t=-\frac{1+u}{v} .
$$

Then, the additive structure $[+]$ on $T$ can be understood as that induced from a comultiplication on the structure ring $\mathbb{Q}[u, v] /\left(u^{2}+u v+v^{2}-1\right)$ of $S$ : See N.Suwa [11] for more thorough generalization of Kummer theory toward contexts of commutative group schemes.

Remark 4.7. If $p \neq q$ and $t=(p-1)(2 q-1) /(p-q)$ lies in the lower half plane $\mathcal{H}^{-}$, then $\mathcal{S}_{p, q}$ alters some positive triangle triples $(a, b, c)$ into non-positive ones, i.e., into triples $\left(a^{\prime}, b^{\prime}, c^{\prime}\right)$ with $\operatorname{Im}\left(\frac{a^{\prime}-b^{\prime}}{c^{\prime}-b^{\prime}}\right)<0$. This case still makes sense and is interesting, however, in this paper we do not focus on non-regular operations. 
Remark 4.8. The above expression (4.1) of our triangle modulus $\phi(\Delta)$ was notified by G.Nicollier to the first author. It is a crucial observation that the triangles on the same radius in $\mathcal{D}$ have the same Brocard angle $\omega(\Delta)$, i.e.,

$$
|\phi(\Delta)|=r^{3}(0<r<1) \Leftrightarrow \frac{\cot (\omega(\Delta))}{\sqrt{3}}=\frac{1+r^{2}}{1-r^{2}} .
$$

See the Introduction of [8]. The quantity inside the cube in 4.1 is called a shape function on triangle triples and has been intensively studied by recent works by M.Hajja et. al. (cf. e.g., [3] and references therein).

\section{Fourier transform $\mathcal{S}\left[\eta, \eta^{\prime}\right]$}

Let $\operatorname{area}(\Delta)$ denote the area of a triangle $\Delta$. We begin this section with:

Proposition 5.1. Suppose $p, q \in \mathbb{R}(p q \neq 1)$. The ratio area $\left(\mathcal{S}_{p, q}(\Delta)\right) /$ area $(\Delta)$ is given by

$$
\frac{\operatorname{area}\left(\mathcal{S}_{p, q}(\Delta)\right)}{\operatorname{area}(\Delta)}=\left|\frac{p-q}{1-p q} \cdot\left(1+t_{p, q} \omega^{2}\right)\right|^{2},
$$

where the quantity $t_{p, q}$ is as in (4.2).

Proof. Since the operation $\mathcal{S}_{p, q}$ belongs to affine geometry, the ratio area $\left(\mathcal{S}_{p, q}(\Delta)\right) / \operatorname{area}(\Delta)$ is independent of choice of $\Delta$. We may assume, for example, $\Delta$ is the equilateral triangle $\Delta_{0}=$ $\left(1, \omega, \omega^{2}\right)$. We make use of G.Nicollier's interpretation of $\mathcal{S}_{p, q}(\Delta)$ in [8] $\S 4$ (Remark in p.76-77): if $p(2 q-3) \neq-1$ then $\mathcal{S}_{p, q}(\Delta) \equiv \frac{p(2 q-3)+1}{1-p q} \Delta * K\left(\frac{q-p}{p(2 q-3)+1}\right)$, where $\equiv$ means congruence up to parallel translations and $\Delta * K(z)$ is (the convolution with the Kiepert triangle $K(z)$ ) the triangle obtained from the three apices of ' $(1-z: z)$-ears' of the sides of $\Delta$. It follows easily that

$$
\frac{\operatorname{area}\left(\Delta_{0} * K(z)\right)}{\operatorname{area}\left(\Delta_{0}\right)}=3\left|z-\left(\frac{1}{2}-\frac{\sqrt{3} i}{6}\right)\right|^{2}
$$

Noting this and the equality $p(2 q-3)+1=\left(2-t_{p, q}\right)(q-p)$, we obtain the desired result by computing the left hand side as

$$
\frac{\operatorname{area}\left(\mathcal{S}_{p, q}\left(\Delta_{0}\right)\right)}{\operatorname{area}\left(\Delta_{0}\right)}=\left|\frac{\left(2-t_{p, q}\right)(p-q)}{(1-p q)}\right|^{2} \cdot 3\left|\frac{1}{2-t_{p, q}}-\left(\frac{1}{2}-\frac{\sqrt{3} i}{6}\right)\right|^{2}=\left|\frac{p-q}{1-p q}\left(1+t_{p, q} \omega^{2}\right)\right|^{2} \text {. }
$$

We may remove the assumption $p(2 q-3) \neq-1$ for the ratio is obviously continuous in parameters $p, q$.

Example 5.3. The famous Routh's theorem states that both $\mathcal{S}_{\frac{1}{3}, \frac{2}{3}}(\Delta)$ and $\mathcal{S}_{\frac{2}{3}, \frac{1}{3}}(\Delta)$ have oneseventh area of $\Delta$. Applying Proposition 5.14 (ii) shown later, we can express matrices for these two Routh operators as $\mathrm{S}_{\frac{1}{3}, \frac{2}{3}}=J \mathcal{S}_{\frac{4}{5}, \frac{2}{3}}=\mathrm{J}^{2} \mathrm{~S}_{\frac{1}{3}, \frac{1}{5}}$ and $\mathcal{S}_{\frac{2}{3}, \frac{1}{3}}=\mathrm{J} \mathcal{S}_{\frac{1}{5}, \frac{1}{3}}=\mathrm{J}^{2} \mathcal{S}_{\frac{2}{3}, \frac{4}{5}}$. The twofold (families of) Routh triangles $\mathcal{S}_{\frac{1}{3}, \frac{2}{3}}(\Delta)$ and $\mathcal{S}_{\frac{2}{3}, \frac{1}{3}}(\Delta)$ share the centroid with $\Delta$, however, they are generally not point-symmetrical to each other. Using Proposition 5.14 (iii), we find that $\mathcal{S}_{p, q}(\Delta)$ and $\mathcal{S}_{q, p}(\Delta)$ form a point-symmetrical pair for every triangle $\Delta$ if and only if $4 p q-$ $3 p-3 q+2=0$. For example, $\left(\mathcal{S}_{\frac{1}{3}, \frac{3}{5}}, \mathcal{S}_{\frac{3}{5}, \frac{1}{3}}\right),\left(\mathcal{S}_{\frac{2}{5}, \frac{4}{7}}, \mathcal{S}_{\frac{4}{7}}, \frac{2}{5}\right)$ are such pairs, but $\left(\mathcal{S}_{\frac{1}{3}, \frac{2}{3}}, \mathcal{S}_{\frac{2}{3}, \frac{1}{3}}\right)$ is not. In [6, Example 2.15], we give another characterization of the Routh operators by their special relationships to "generalized median operators". The above area formula for real parameters $p, q$ in Proposition 5.1 may be regarded as a special case of a more general formula given in [1], where other interesting numerical examples with rational parameters can be found. Generalization for complex parameters will be discussed in Proposition 5.12 below. 
Motivated by Proposition 5.1, let us introduce new quantities $\eta_{p, q}$ and $\eta_{p, q}^{\prime}$ by:

$$
\eta_{p, q}:=\left(1+t_{p, q} \omega\right) \frac{p-q}{1-p q}, \quad \eta_{p, q}^{\prime}:=\left(1+t_{p, q} \omega^{2}\right) \frac{p-q}{1-p q} .
$$

It is clear that the parameter $\xi_{p, q}$ introduced in 4.2 satisfies $\xi_{p, q}=\eta_{p, q} / \eta_{p, q}^{\prime}$. Recall that the parameter $\xi_{p, q}$ comes from the action $T_{p, q}$ on the moduli disk $\mathcal{D}$, the moduli disk for the similarity classes of triangle. The above numerator-denominator separation $\left(\eta_{p, q}, \eta_{p, q}^{\prime}\right)$ of the moduli action $\xi_{p, q}$ does, in fact, recover the operator $\mathcal{S}_{p, q}$ itself as follows:

Proposition 5.5. Notations being (2.1), the operator $\mathcal{S}_{p, q}$ is determined by the parameter $\left(\eta_{p, q}, \eta_{p, q}^{\prime}\right)$ with

$$
\begin{aligned}
\alpha_{p, q} & =\frac{1}{3}\left(1+\eta_{p, q}+\eta_{p, q}^{\prime}\right), \\
\beta_{p, q} & =\frac{1}{3}\left(1+\omega \eta_{p, q}+\omega^{2} \eta_{p, q}^{\prime}\right), \\
\gamma_{p, q} & =\frac{1}{3}\left(1+\omega^{2} \eta_{p, q}+\omega \eta_{p, q}^{\prime}\right) .
\end{aligned}
$$

Proof. This follows immediately after substituting $p=p\left(\eta, \eta^{\prime}\right), q=q\left(\eta, \eta^{\prime}\right)$ for $\alpha_{p, q}, \beta_{p, q}$ and $\gamma_{p, q}$ in 2.1.

Now, we shall give interpretation of the parameter $\left(\eta_{p, q}, \eta_{p, q}^{\prime}\right)$ from the view point of finite Fourier series. In the spirit of [10, [8], considering a triangle triple $\Delta=(a, b, c)$ is equivalent to considering its Fourier transform $\left(\psi_{0}, \psi_{1}, \psi_{2}\right)$ defined as the coefficients of a quadratic polynomial $\Psi_{\Delta}(T)=\psi_{0}(\Delta)+\psi_{1}(\Delta) T+\psi_{2}(\Delta) T^{2} \in \mathbb{C}[T]$ uniquely determined by the identities $\Psi_{\Delta}(1)=$ $a, \Psi_{\Delta}(\omega)=b, \Psi_{\Delta}\left(\omega^{2}\right)=c$. Explicitly, they are given by $\psi_{0}(\Delta)=\frac{1}{3}(a+b+c), \psi_{1}(\Delta)=$ $\frac{1}{3}\left(a+b \omega^{2}+c \omega\right), \psi_{2}(\Delta)=\frac{1}{3}\left(a+b \omega+c \omega^{2}\right)$.

Definition 5.6. Let $\Delta=(a, b, c)$ be a triangle triple. Notations being as above, the Fourier transformed vector $\psi(\Delta)$ of $\Delta$ is defined by

$$
\psi(\Delta)=\left(\begin{array}{c}
\psi_{0}(\Delta) \\
\psi_{1}(\Delta) \\
\psi_{2}(\Delta)
\end{array}\right)
$$

By simple (matrix) computation, we find that the parameter $\left(\eta_{p, q}, \eta_{p, q}^{\prime}\right)$ is just the ratio of the 2nd and 3rd Fourier coefficients. More precisely

Proposition 5.7. For each triangle triple $\Delta$, it holds that

$$
\psi\left(\mathcal{S}_{p, q}(\Delta)\right)=\operatorname{diag}\left(1, \eta_{p, q}^{\prime}, \eta_{p, q}\right) \cdot \psi(\Delta) .
$$

Proof. The asserted identity is equivalent to

$$
\mathrm{S}_{p, q}=W\left(\begin{array}{ccc}
1 & 0 & 0 \\
0 & \eta_{p, q}^{\prime} & 0 \\
0 & 0 & \eta_{p, q}
\end{array}\right) W^{-1} \quad \text { with } \quad W=\left(\begin{array}{ccc}
1 & 1 & 1 \\
1 & \omega & \omega^{2} \\
1 & \omega^{2} & \omega
\end{array}\right)
$$

in the space of $3 \times 3$-matrices.

Observing the above simple form of $\mathcal{S}_{p, q}$ acting on the Fourier transformed triangle triples, we are led to the idea of viewing the operation $\mathcal{S}_{p, q}$ by the parameters $\left(\eta, \eta^{\prime}\right) \in \mathbb{C}^{2}$ rather than the original parameter $(p, q) \in \mathbb{C}^{2}$ with $p q \neq 1$ :

Definition 5.9. For $\left(\eta, \eta^{\prime}\right) \in \mathbb{C}^{2}$, we define the operator $\mathcal{S}\left[\eta, \eta^{\prime}\right]$ on the triangle triples by the action on their Fourier transform vectors, and define its corresponding matrix $\mathrm{S}\left[\eta, \eta^{\prime}\right]$ as

$$
\psi\left(\mathcal{S}\left[\eta, \eta^{\prime}\right](\Delta)\right)=\left(\begin{array}{ccc}
1 & 0 & 0 \\
0 & \eta^{\prime} & 0 \\
0 & 0 & \eta
\end{array}\right) \psi(\Delta), \quad \mathrm{S}\left[\eta, \eta^{\prime}\right]:=W\left(\begin{array}{ccc}
1 & 0 & 0 \\
0 & \eta^{\prime} & 0 \\
0 & 0 & \eta
\end{array}\right) W^{-1}
$$


where $W$ is the Vandermonde matrix used in (5.8).

Remark 5.11. It is now evident that the collection

$$
\mathbb{S}:=\left\{\mathrm{S}\left[\eta, \eta^{\prime}\right] \mid \eta, \eta^{\prime} \in \mathbb{C}\right\}=\left\{\alpha \mathbf{I}+\beta \mathrm{J}+\gamma \mathrm{J}^{2} \mid \alpha+\beta+\gamma=1\right\}
$$

forms a multiplicative submonoid of $M_{3}(\mathbb{C})$ containing the set $\mathbb{S}_{p, q}$ considered in Note 3.8 . Let $\mathbb{S}^{\times}:=\left\{\mathrm{S}\left[\eta, \eta^{\prime}\right] \mid \eta, \eta^{\prime} \in \mathbb{C}^{\times}\right\}$be the set of invertible elements of $\mathbb{S}$ which forms an abelian subgroup isomorphic to $\left(\mathbb{C}^{\times}\right)^{2}$ in $\mathrm{GL}_{3}(\mathbb{C})$. Note that $\mathbb{S}_{p, q} \subset \mathbb{S} \supset \mathbb{S}^{\times}$. In the special case when $\left(p_{1}, q_{1}\right)=\left(\frac{1}{3}, \frac{1}{4}\right),\left(p_{2}, q_{2}\right)=\left(-\frac{7}{8},-\frac{1}{9}\right), \lambda_{1}=\lambda_{2}+2 \lambda_{3}=0$ discussed in loc. cit. we can take the corresponding parameters $\left(\xi_{1}, \eta_{1}, \eta_{1}^{\prime}\right)=\left(-\frac{1+4 \omega}{3+4 \omega}, \frac{1+4 \omega}{11},-\frac{3+4 \omega}{11}\right),\left(\xi_{2}, \eta_{2}, \eta_{2}^{\prime}\right)=\left(\frac{3 \omega-1}{3 \omega^{2}-1}, \frac{11}{13}(3 \omega-\right.$ 1), $\left.\frac{11}{13}\left(3 \omega^{2}-1\right)\right)$. This shows that $S_{p_{1}, q_{1}}, S_{p_{2}, q_{2}} \in \mathbb{S}^{\times} \cap \mathbb{S}_{p, q}$. Accordingly, the composition $\mathrm{S}_{p_{1}, q_{1}} \mathrm{~S}_{p_{2}, q_{2}}=\mathrm{S}\left[\eta_{1}, \eta_{1}^{\prime}\right] \mathrm{S}\left[\eta_{2}, \eta_{2}^{\prime}\right]=\mathrm{S}\left[\eta_{1} \eta_{2}, \eta_{1}^{\prime} \eta_{2}^{\prime}\right]=\mathrm{S}\left[\omega^{2}, \omega\right]$ lies in $\mathbb{S}^{\times}$, whereas it is not available in $\mathbb{S}_{p, q}$ as discussed in Note 3.8 .

We now generalize Proposition 5.1 for arbitrary complex parameters.

Proposition 5.12. Let $\Delta=(a, b, c)$ be a triangle triple with its Fourier transform $\psi(\Delta)=$ $\left(\psi_{0}, \psi_{1}, \psi_{2}\right)$, and let $\left(\eta, \eta^{\prime}\right) \in \mathbb{C}^{2}$. Then,

(i) $\operatorname{area}(\Delta)=\left.\frac{3 \sqrt{3}}{4}|| \psi_{1}\right|^{2}-\left|\psi_{2}\right|^{2} \mid$.

(ii) If $\Delta$ is non-degenerate, then

$$
\frac{\operatorname{area}\left(\mathcal{S}\left[\eta, \eta^{\prime}\right](\Delta)\right)}{\operatorname{area}(\Delta)}=\left|\frac{\left|\eta^{\prime} \psi_{1}\right|^{2}-\left|\eta \psi_{2}\right|^{2}}{\left|\psi_{1}\right|^{2}-\left|\psi_{2}\right|^{2}}\right|
$$

(iii) The operator $\mathcal{S}\left[\eta, \eta^{\prime}\right]$ preserves areas of triangles if and only if $|\eta|=\left|\eta^{\prime}\right|=1$.

Proof. (i). Recall that area $(\Delta)=\mid \operatorname{Im}((b-a) \overline{(c-a)} \mid / 2$. In terms of $\psi(\Delta)$, one can express

$$
(b-a) \overline{(c-a)}=(\omega-1)^{2}\left|\psi_{1}\right|^{2}+(\bar{\omega}-1)^{2}\left|\psi_{2}\right|^{2}+(\omega-1)(\bar{\omega}-1)\left(\psi_{1} \overline{\psi_{2}}+\psi_{2} \overline{\psi_{1}}\right) .
$$

Noting that the last term is real and that $\operatorname{Im}\left((\omega-1)^{2}\right)=-3 \sqrt{3} / 2$, we obtain the assertion. (ii) follows from (i) and Definition 5.9. (iii) First, consider special cases of positive and negative equilateral triangles $\Delta_{0}=\left(1, \omega, \omega^{2}\right)$ and $\Delta_{1}=\left(1, \omega^{2}, \omega\right)$, where $\psi\left(\Delta_{0}\right)=(0,1,0), \psi\left(\Delta_{1}\right)=$ $(0,0,1)$. By virtue of (ii), area $\left(\left(\mathcal{S}\left[\eta, \eta^{\prime}\right]\right)\left(\Delta_{i}\right)\right)=\operatorname{area}\left(\Delta_{i}\right)$ for $i=0,1$ imply $\left|\eta^{\prime}\right|=1,|\eta|=1$ respectively. Conversely, if $|\eta|=\left|\eta^{\prime}\right|=1$, then it is easy to see from (ii) that $\mathcal{S}\left[\eta, \eta^{\prime}\right]$ preserves areas of every triangle.

Before going further, let us quickly summarize how the parameters $p, q$ can be recovered from $\eta=\eta_{p, q}, \eta^{\prime}=\eta_{p, q}^{\prime}$ :

Proposition 5.13. Define two rational functions $p\left(y, y^{\prime}\right), q\left(y, y^{\prime}\right) \in \mathbb{C}\left(y, y^{\prime}\right)$ by

$$
p\left(y, y^{\prime}\right)=\frac{1+y+y^{\prime}}{2-\omega y-\omega^{2} y^{\prime}}, \quad q\left(y, y^{\prime}\right)=\frac{1+\omega y+\omega^{2} y^{\prime}}{2-y-y^{\prime}} .
$$

Then, for $\eta=\eta_{p, q}$ and $\eta^{\prime}=\eta_{p, q}^{\prime}$, it holds that

$$
\mathcal{S}_{p, q}=\mathcal{S}_{p\left(\eta, \eta^{\prime}\right), q\left(\eta, \eta^{\prime}\right)}=\mathcal{S}\left[\eta, \eta^{\prime}\right]
$$

as long as involved quantities are well-defined. Moreover, if $p_{i}=p\left(\eta_{i}, \eta_{i}^{\prime}\right), q_{i}=q\left(\eta_{i}, \eta_{i}^{\prime}\right)(i=$ $1,2,3)$ are given with $\eta_{1} \eta_{2}=\eta_{3}, \eta_{1}^{\prime} \eta_{2}^{\prime}=\eta_{3}^{\prime}$, then $\mathcal{S}_{p_{1}, q_{1}} \circ \mathcal{S}_{p_{2}, q_{2}}=\mathcal{S}_{p_{3}, q_{3}}$ and vice versa.

Proof. The first statement follows by straightforward computations solving the equations 4.2 and (5.4) reversely. The latter statement can be seen from the above formula (5.10). Or, more directly, it follows from the fact that the cyclic matrices $\mathrm{S}_{p, q} \in \mathbb{C l}+\mathbb{C J}+\mathbb{C J}{ }^{2}$ are simultaneously diagonalized to $\operatorname{diag}\left(1, \eta_{p, q}^{\prime}, \eta_{p, q}\right)$ (via base change by the Vandermonde matrix $\left.\left(\omega^{i-1, j-1}\right)_{i, j}\right)$. 
The following matrix properties are often useful to interpret relations between operators $\mathcal{S}_{p, q}$ geometrically.

Proposition 5.14. Below, quantities $p, q \in \mathbb{C}$ are understood to be taken so that involved rational functions and operators are defined.

(i) $\mathrm{S}_{p, q}=\mathrm{S}\left[\eta, \eta^{\prime}\right]$ if and only if $\mathrm{S}_{q, p}=\mathrm{S}\left[\eta^{\prime} \omega^{2}, \eta \omega\right]=\mathrm{JS}\left[\eta^{\prime}, \eta\right]$.

(ii) $\mathrm{S}_{p, q}=\mathrm{JS}_{p_{1}, q_{1}}=\mathrm{J}^{2} \mathrm{~S}_{p_{2}, q_{2}}$,

where $\left(p_{1}, q_{1}\right)=\left(\frac{q(p-1)}{2 p q-p-q}, 1-p\right),\left(p_{2}, q_{2}\right)=\left(1-q, \frac{p(q-1)}{2 p q-p-q}\right)$.

(iii) $\mathrm{S}_{p, q}+\mathrm{S}_{p^{\prime}, q^{\prime}}=\frac{2}{3}\left(\mathrm{I}+\mathrm{J}+\mathrm{J}^{2}\right)$, where $\left(p^{\prime}, q^{\prime}\right)=\left(\frac{2-3 p+p q}{1+3 q-4 p q}, \frac{2-3 q+p q}{1+3 p-4 p q}\right)$.

Proof. (i) follows from the observation in Proposition 5.13 that $p\left(y, y^{\prime}\right)$ and $q\left(y, y^{\prime}\right)$ are interchanged to each other when $\left(y, y^{\prime}\right)$ is replaced by $\left(\omega^{2} y^{\prime}, \omega y\right)$. The last equality

$$
\mathrm{S}\left[\eta^{\prime} \omega^{2}, \eta \omega\right]=\mathrm{JS}\left[\eta^{\prime}, \eta\right]
$$

follows by a simple matrix computation (cf. [6, (2.1)]). (ii) is just a translation of (5.15) in terms of the original parameters $(p, q)$. (iii): The variable $\left(p^{\prime}, q^{\prime}\right)$ is defined so that $\mathrm{S}_{p, q}=\mathrm{S}\left[\eta, \eta^{\prime}\right]$ and $\mathrm{S}_{p^{\prime}, q^{\prime}}=\mathrm{S}\left[-\eta,-\eta^{\prime}\right]$. The identity reflects a simple consequence of $\frac{1}{2}\left(\mathrm{~S}\left[\eta, \eta^{\prime}\right]+\mathrm{S}\left[-\eta,-\eta^{\prime}\right]\right)=$ $W \operatorname{diag}(1,0,0) W^{-1}=\frac{1}{3}\left(\mathrm{I}+\mathrm{J}+\mathrm{J}^{2}\right)$.

Example 5.16. In the following table, we summarize examples of $\mathcal{S}_{p, q}$ with special parameters $p, q, \xi_{p, q}, \eta_{p, q}$ and $\eta_{p, q}^{\prime}$ :

\begin{tabular}{|c|c|c|c|c|c|}
\hline$p$ & $q$ & $\xi_{p, q}$ & $\eta_{p, q}$ & $\eta_{p, q}^{\prime}$ & $\mathcal{S}_{p, q}$ \\
\hline 1 & $*(\neq 1)$ & 1 & 1 & 1 & 3.2 : identity \\
\hline$*(\neq 1)$ & 1 & $\omega$ & $\omega^{2}$ & $\omega$ & 3.2 : cyclic permutation \\
\hline 0 & 0 & $\omega^{2}$ & $\omega$ & $\omega^{2}$ & (3.2) : cyclic permutation \\
\hline 0 & $1-s$ & $\frac{s+(1-s) \omega}{s \omega+(1-s)}$ & $s \omega+(1-s) \omega^{2}$ & $s \omega^{2}+(1-s) \omega$ & $\operatorname{Ex}[2.4: s-m e d i a l([3,[7])$ \\
\hline$s$ & $1-s$ & $\frac{\omega+s}{1+\omega s}$ & $\frac{1-2 s}{1-s+s^{2}}\left(\omega^{2}+\omega s\right)$ & $\frac{1-2 s}{1-s+s^{2}}\left(\omega+\omega^{2} s\right)$ & Ex 2.4: s-Routh $([3,[7])$ \\
\hline$\frac{2 s}{s+3}$ & $\frac{s}{2 s-3}$ & $\frac{s+\omega}{s+\omega^{2}}$ & $s+\omega$ & $s+\omega^{2}$ & Ex 2.4: $s$-median $([\underline{3},[\underline{6}])$ \\
\hline 0 & $\frac{1-\omega^{2}}{3}$ & 0 & 0 & -1 & Ex 2.5: Napoleon \\
\hline 0 & $\frac{1-\omega}{3}$ & $\infty$ & -1 & 0 & Ex 2.5: Napoleon (reversed) \\
\hline$r(\neq 2)$ & $\frac{1}{2}$ & 1 & $\frac{1-2 r}{r-2}$ & $\frac{1-2 r}{r-2}$ & Prop. 4.4 (iii) \\
\hline$\frac{1}{2}$ & $r(\neq 2)$ & $\omega$ & $\frac{1-2 r}{r-2} \omega^{2}$ & $\frac{1-2 r}{r-2} \omega$ & Prop. 4.4 (iii) \\
\hline$q(\neq-1)$ & $q(\neq-1)$ & $\omega^{2}$ & $\frac{1-2 q}{1+q} \omega$ & $\frac{1-2 q}{1+q} \omega^{2}$ & Prop. 4.4 (iii) \\
\hline$\frac{1+2 e^{i \theta}}{2+e^{i \theta}}$ & $\frac{1}{2}$ & 1 & $e^{i \theta}$ & $e^{i \theta}$ & $\theta$-rotation \\
\hline
\end{tabular}

\section{Area preserving operators $\mathcal{S}_{\mathrm{ap}}\left(\theta_{x}, \theta_{y}, \theta_{y}^{\prime}\right)$}

We once again recall the notations $\rho:=e^{2 \pi i / 6}, \omega:=e^{2 \pi i / 3}$. Given $\mathcal{S}_{p, q}$, we have the secondary parameters $\left(\xi_{p, q}, \eta_{p, q}, \eta_{p, q}^{\prime}\right)$ with $\eta_{p, q}=\xi_{p, q} \eta_{p, q}^{\prime}$ as studied in the preceding sections. If $\left|\xi_{p, q}\right|=1$, then it operates on the moduli disc of similarity classes by a bijective rotation, and if $\left|\eta_{p, q}\right|=$ $\left|\eta_{p, q}^{\prime}\right|=1$, then area $\left(\mathcal{S}_{p, q}(\Delta)\right)=\operatorname{area}(\Delta)$ for every triangle triple $\Delta$ (cf. 44.3), Proposition 5.12).

We begin with:

Proposition 6.1. The operator $\mathcal{S}_{p, q}$ has a finite period $N$ if and only if $\xi_{p, q}^{N}=\eta_{p, q}^{N}=\eta_{p, q}^{\prime}=1$, In particular, such $\mathcal{S}_{p, q}$ is normal and preserves areas of triangles.

Proof. This follows immediately from our discussions above and in $\S 5$. 
Definition 6.2. We call $\mathcal{S}_{p, q}$ an area-preserving normal operator if the associated parameters satisfy $\left|\xi_{p, q}\right|=\left|\eta_{p, q}\right|=\left|\eta_{p, q}^{\prime}\right|=1$.

In Proposition 5.13, we considered two rational functions $p\left(y, y^{\prime}\right), q\left(y, y^{\prime}\right) \in \mathbb{C}\left(y, y^{\prime}\right)$ that represent the parameter $(p, q)$ by the new parameter $\left(\eta, \eta^{\prime}\right)$. This is natural because $\eta, \eta^{\prime}$ are eigenvalues of $\mathcal{S}_{p, q}$. Meanwhile, when we restrict our attention to the area-preserving operators, we find the parameter $\left(\xi=\eta / \eta^{\prime}, \eta\right)$ still deserves to be utilized. So we shall define two rational functions $\mathbf{p}(x, y), \mathbf{q}(x, y)$ by

$$
\begin{aligned}
& \mathbf{p}(x, y):=p\left(y, \frac{y}{x}\right)=\rho \cdot \frac{x y+x+y}{x y+2 \rho x+\rho^{2} y}, \\
& \mathbf{q}(x, y):=q\left(y, \frac{y}{x}\right)=\rho^{-1} \cdot \frac{x y-\rho x-\rho^{-1} y}{x y-2 x+y} .
\end{aligned}
$$

In fact, we find the following algebraic functional equations

$$
\begin{aligned}
& \mathbf{p}(x, y)=\mathbf{q}\left(\omega x^{-1}, \omega^{2} x^{-1} y\right), \quad \mathbf{q}(x, y)=\mathbf{p}\left(\omega x^{-1}, \omega^{2} x^{-1} y\right) \\
& \mathbf{p}(y, x)=\frac{\mathbf{p}(x, y)(\mathbf{q}(x, y)-1)}{\rho(\mathbf{p}(x, y)-1) \mathbf{q}(x, y)-(\mathbf{p}(x, y)-\mathbf{q}(x, y))} \\
& \mathbf{q}(y, x)=\frac{\mathbf{q}(x, y)-1}{(1+\rho) \mathbf{q}(x, y)-1} .
\end{aligned}
$$

Moreover, on the normal area-preserving torus

$$
\mathcal{T}:=S^{1} \times S^{1}=\{(x, y) \in \mathbb{C} ;|x|=|y|=1\},
$$

we have a closer look at the functions $\mathbf{p}(x, y)$ and $\mathbf{q}(x, y)$ with their extra-symmetry properties: namely, if we consider $\mathbf{p}(x, y)$ and $\mathbf{q}(x, y)$ as $\mathbb{C}$-valued partial functions on $\mathcal{T}$, then,

(i) $\mathbf{p}(x, y)$ is defined on $\mathcal{T}-\left\{\left(\omega, \omega^{2}\right)\right\}$.

(ii) $\mathbf{q}(x, y)$ is defined on $\mathcal{T}-\{(1,1)\}$.

On these regions of definition, they have functional equations:

$$
\mathbf{p}(x, y)+\overline{\mathbf{p}\left(\omega^{2} x^{-1}, \omega y^{-1}\right)}=1, \quad \mathbf{q}(x, y)+\overline{\mathbf{q}\left(x^{-1}, y^{-1}\right)}=1 .
$$

The above functional equations $(6.5)-(6.9)$ can be verified by straightforward calculations (assisted by symbolic algebraic system, once they are found).

One possible way to see a source reason behind the above symmetric formulas may be connecting $\mathbf{q}(x, y)$ with the following simple rational function

$$
R(x, y):=\frac{2 x y-x-y}{\sqrt{3}(y-x)} .
$$

In fact, one can interpret a modified function

$$
Q(x, y):=i\left(\mathbf{q}(x, y)-\frac{1}{2}\right) \quad \text { as to be: } \quad Q(x, y)=\frac{1}{2}\left(\frac{\sqrt{3} R(x, y)-1}{R(x, y)+\sqrt{3}}\right) .
$$

The identity (6.7) and the first formula of $(6.9)$ are consequences of the obvious symmetry:

$$
R(x, y)+R(y, x)=0, \quad R\left(x^{-1}, y^{-1}\right)=\overline{R(x, y)} \quad(|x|=|y|=1),
$$

while 6.6) and the second formula of 6.9 are their translations through 6.5).

Corollary 6.13. The values of $\mathbf{q}(x, y)$ on $\mathcal{T}$ are determined from those values $\mathbf{q}\left(e^{i \theta}, e^{i \phi}\right)$ on the region $\mathcal{T}_{0}:=\{(\theta, \phi) \mid 0<\theta<2 \pi, 0 \leq \phi \leq \theta, \theta+\phi \leq 2 \pi\}$.

Note that the identity 6.5 tells how the function $\mathbf{q}(x, y)$ determines $\mathbf{p}(x, y)$. 
Proof. This follows from observing values of $R(x, y)$ and the symmetric relations (6.12): The values of $R\left(e^{i \theta}, e^{i \phi}\right)$ are determined on $\mathcal{T}_{0}$. Note that on the boundary of $\mathcal{T}_{0}$, the value of $R(x, y)$ is undetermined only at $\theta=\phi=0$; elsewhere it takes $\frac{i}{\sqrt{3}} \tan \frac{\theta}{2}$ on $\{(\theta, \phi) \mid 0<\phi=2 \pi-\theta \leq \pi\}$, constant $1 / \sqrt{3}$ on $\{(\theta, 0) \mid 0<\theta<2 \pi\}$ and $\infty$ on $\{(\theta, \phi) \mid 0<\theta=\phi \leq \pi\}$.

At the end of this article, let us give several illustrations on normal area-preserving operations on triangles. For convenience, let us identify the normal area-preserving torus as:

$$
\mathcal{T} \cong\left\{\left(\theta_{x}, \theta_{y}, \theta_{y}^{\prime}\right) \in(\mathbb{R} / \mathbb{Z})^{3} \mid \theta_{x}=\theta_{y}-\theta_{y}^{\prime}\right\} .
$$

$\operatorname{via}(x, y) \leftrightarrow\left(e^{2 \pi i \theta_{x}}, e^{2 \pi i \theta_{y}}\right)$

Definition 6.14. Define

$$
\mathcal{S}_{\text {ap }}\left(\theta_{x}, \theta_{y}, \theta_{y}^{\prime}\right):=\mathcal{S}\left[e^{2 \pi i \theta_{y}}, e^{2 \pi i \theta_{y}^{\prime}}\right] .
$$

Example 6.15. Here are two orbits of area-preserving operators $\mathcal{S}_{\text {ap }}\left(\theta_{x}, \theta_{y}, \theta_{y}-\theta_{x}\right)$ of order 20 and 28 starting from the initial triangle $\Delta=(0,1,0.7+0.8 i)$ :
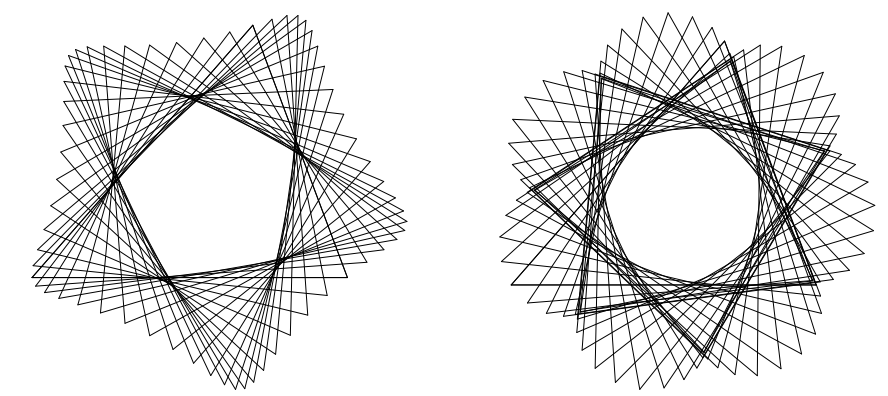

$$
\left\{\mathcal{S}_{\text {ap }}\left(\frac{1}{4}, \frac{1}{5}, \frac{1}{5}-\frac{1}{4}\right)^{n}(\Delta)\right\}_{n \in \mathbb{Z}} \quad\left\{\mathcal{S}_{\text {ap }}\left(\frac{1}{4}, \frac{1}{7}, \frac{1}{7}-\frac{1}{4}\right)^{n}(\Delta)\right\}_{n \in \mathbb{Z}}
$$

Example 6.16. Simple rotations through the angle $\theta$ are realized by $\mathcal{S}_{\text {ap }}(0, \theta, \theta)$. Here are two examples starting from $\Delta=(0,1,0.7+0.3 i)$ :

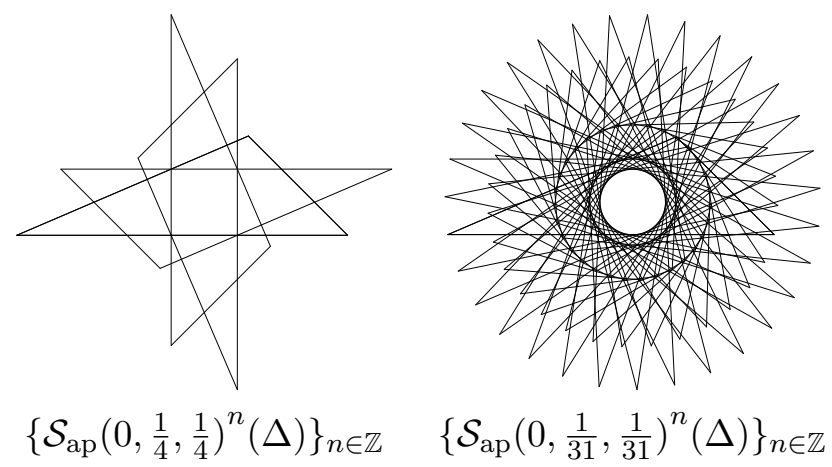

Example 6.17. Trefoil-looking figure appears in the orbits of $\mathcal{S}_{\text {ap }}(\theta, 0,-\theta)$. Starting from the initial triangle $\Delta=(0,1,0.7+0.3 i)$, we have the following two sample illustrations: 

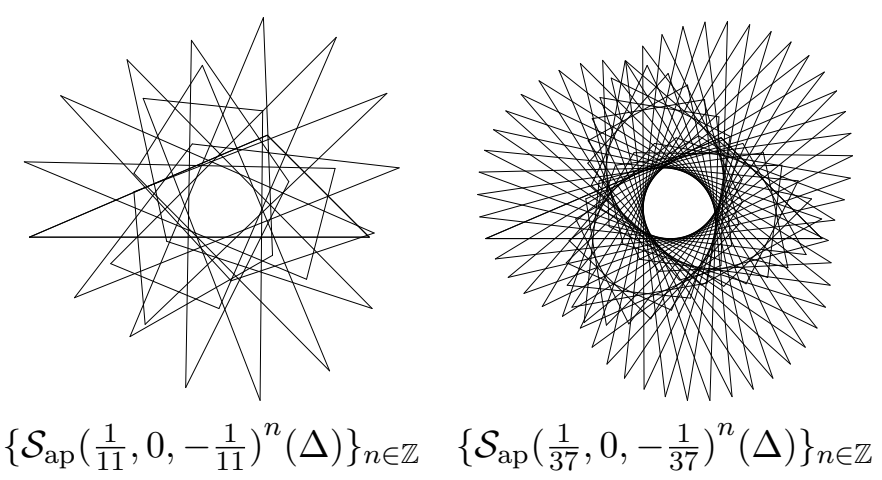

Example 6.18. Here are two orbits of $\mathcal{S}_{\mathrm{ap}}(\theta, \theta, 0)$-images starting from $\Delta=(0,1,0.7+0.5 i)$ in the cases $\theta=\frac{1}{5}, \frac{1}{31}$.
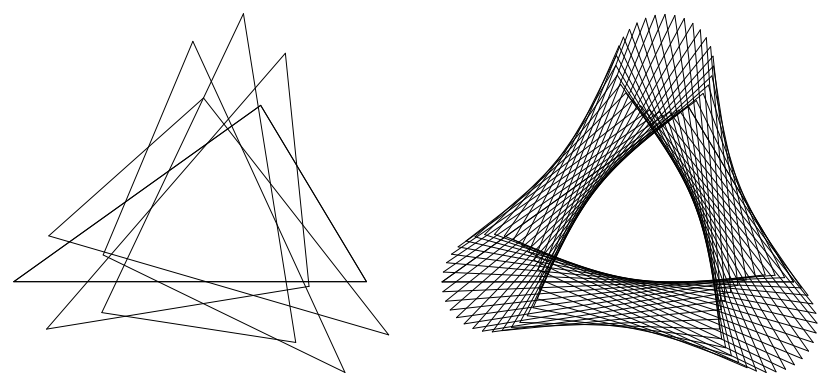

$$
\left\{\mathcal{S}_{\text {ap }}\left(\frac{1}{5}, \frac{1}{5}, 0\right)^{n}(\Delta)\right\}_{n \in \mathbb{Z}} \quad\left\{\mathcal{S}_{\text {ap }}\left(\frac{1}{31}, \frac{1}{31}, 0\right)^{n}(\Delta)\right\}_{n \in \mathbb{Z}}
$$

\section{References}

[1] Á.Bényi, B.Ćurgus, A generalization of Routh's triangle theorem, Amer. Math. Monthly 120 (2013), 841846.

[2] Á.Bényi, B.Ćurgus, Triangles and groups of cevians, J. of Geometry 103 (2012), 375-408.

[3] M. Hajja, On nested sequences of triangles, Result. Math. 54 (2009), 289-299.

[4] T. Kanesaka, Study on Brocard angles and the moduli disk of triangles (in Japanese), Master Thesis at Okayama University, February 2015.

[5] T. Komatsu, Arithmetic of Rikuna's generic cyclic polynomial and generalization of Kummer theory, manuscripta math. 114 (2004), 265-279.

[6] H.Nakamura, H.Ogawa, On generalized median triangles and tracing orbits, Preprint 2019, arXiv:1905.07225 [math.MG]

[7] H.Nakamura, K.Oguiso, Elementary moduli spaces of triangles and iterative processes, J. Math. Sci. Univ. Tokyo 10 (2003), 209-224.

[8] G. Nicollier, Convolution filters for triangles, Forum Geometricorum 13 (2013), 61-85.

[9] H.Ogawa, Quadratic reduction of multiplicative group and its applications (in Japanese), RIMS Kokyuroku 1324 (2003), 217-224.

[10] I.J.Schoenberg, The finite Fourier series and elementary geometry, Amer. Math. Monthly, 57 (1950), 390404.

[11] N.Suwa, Twisted Kummer and Kummer-Artin-Schreier theories, Tohoku Math. J. 60 (2008), 183-218.

Department of Mathematics, Graduate School of Science, Osaka University, Toyonaka, Osaka 560-0043, JAPAN

E-mail address: nakamura@math.sci.osaka-u.ac.jp

Department of Mathematics, Graduate School of Science, Osaka University, Toyonaka, Osaka 560-0043, JAPAN

E-mail address: ogawa@math.sci.osaka-u.ac.jp 